ISSN: 0210-1696

DOI: http://dx.doi.org/10.14201/scero20164744556

\title{
LOS EVENTOS CONTEXTUALES \\ ASOCIADOS A LA OCURRENCIA \\ DE LAS CONDUCTAS PROBLEMÁTICAS \\ EN ENTORNOS ESCOLARES
}

\section{Contextual events related to the occurrence of challenging behavior in educational settings}

\author{
Alba Cortina SERra \\ AMPANS. Manresa. Barcelona \\ University of Vic. C/ Sagrada Familia, 7. 08500 Vic \\ acortina@ampans.cat \\ David Simó Pinatella \\ AMPANS. Manresa. Barcelona \\ Universitat Ramon Llull. Facultat de Psicologia, Ciències de l'Educació i de l'Esport. \\ Blanquerna. Barcelona \\ Cristina Mumbardó AdAm \\ Universitat Ramon Llull. Facultat de Psicologia, Ciències de l'Educació i de l'Esport. \\ Blanquerna. Barcelona \\ Marga Güell Gubianas \\ AMPANS. Manresa. Barcelona \\ Edwin JONES \\ University Health Board. Service Development Consultant at Abertawe Bro, \\ Morgannwg. Reino Unido \\ Kathy Lowe \\ University Health Board. Service Development Consultant at Abertawe Bro, Morgannwg. \\ Reino Unido
}

Recepción: 1 de abril de 2016

Fecha de aceptación definitiva: 25 de noviembre de 2016 
RESUMEN: Las conductas problemáticas que presentan las personas con discapacidad intelectual (DI) influyen negativamente en su calidad de vida. Los principios derivados del análisis conductual aplicado subrayan la relación existente entre dichas conductas y el entorno donde se desarrollan. Este estudio explora aquellas variables de antecedentes relacionadas con la ocurrencia de las conductas problemáticas que presentan los alumnos de primaria de una escuela de educación especial mediante un instrumento de evaluación funcional indirecta: el "Inventario de Evaluación del Contexto". Un total de 17 alumnos participaron en el estudio en el que se evaluaron 25 conductas problemáticas. Los resultados identifican las variables "sociales/culturales" y las de la "naturaleza de la tarea o la actividad" como aquellas más asociadas a la ocurrencia de las conductas problemáticas. Finalmente, se discuten los resultados del artículo en relación a sus implicaciones prácticas y de cara a futuras investigaciones, subrayando la importancia de la prevención y la creación de entornos educativos universales.

Palabras Clave: conductas problemáticas; eventos contextuales; evaluación funcional; discapacidad intelectual; Inventario de Evaluación del Contexto.

AвSTRACT: Challenging behaviors (CB) displayed by people with intellectual disabilities (ID) have a negative effect on their quality of life. The operant theory principles emphasize the relationship between these behaviors and the environment where they take place. This study aimed to identify the contextual variables that are closely related to the occurrence of $\mathrm{CB}$ exhibited by children who attend a special school by using an indirect functional assessment instrument; the Contextual Assessment Inventory. Therefore, 17 students have participated in this study where a total of 25 behaviors have been assessed. The results suggested that the social/cultural and the nature of the task or activity categories were more strongly associated with the occurrence of CB. Finally, results are discussed in terms of implications for practitioners. Future researches might address the importance of prevention and universal educational systems.

KEYWORDS: problem behavior; contextual events; functional assessment; intellectual disability; Contextual Assessment Inventory.

\section{Introducción}

$\mathrm{L}$

AS CONDUCTAS PROBLEMÁTICAS QUE PRESENTAN LOS ALUMNOS con discapacidad intelectual (DI) suelen ser la principal fuente de alteración de las prácticas educativas en los centros escolares (Moreno, 2011; Sugai y Horner, 2002). Las conductas problemáticas no solo se entienden como una barrera a la inclusión y participación en la comunidad (Barrat, McGill y Hugher, 2012), sino que aquellos alumnos que presentan dichas conductas suelen tener mayores dificultades en las interacciones con sus compañeros o familiares y más probabilidades de ser expulsados del centro educativo (Crone y Horner, 2003). Además, las conductas problemáticas pueden ser identificadas como un factor estresante para el profesional que los atiende (Lecavalier, Leone y Wiltz, 2006; Ross, Romer y Horner, 2011) y, como consecuencia, pueden generar actitudes negativas por parte de los profesionales.

(C) Ediciones Universidad de Salamanca / CC BY-NC-ND

Siglo Cero, vol. 47 (4), n. ${ }^{\circ}$ 260, 2016, octubre-diciembre, pp. 45-56

$$
-46-
$$


Aunque tradicionalmente se han utilizado estrategias reactivas en el tratamiento de las conductas problemáticas en entornos educativos (Sugai y Horner, 2002), numerosas investigaciones evidencian el uso de estrategias preventivas para dar respuesta a dichas conductas (p. e., Miller, Dufrene, Sterling, Olmi y Bachmayer, 2015; SimóPinatella, Alomar-Kurz, Font-Roura, Giné y McGill, 2015). Desde esta perspectiva, el Apoyo Conductual Positivo (ACP) se plantea como un modelo de evaluación e intervención preventivo que enfatiza la enseñanza de conductas sociales adecuadas (Carr et al., 2002), así como la creación y promoción de sistemas y entornos positivos que sean sostenibles (Bradshaw, Reinke, Brown, Bevans y Leaf, 2008). Para ello, el ACP hace uso de un conjunto de estrategias, de menor a mayor intensidad, con el fin de dar respuesta a las necesidades conductuales del alumnado (Stormont, Lewis, Beckner y Johnson, 2008) y mejorar el clima de convivencia de los centros educativos (Lassen, Steele y Sailor, 2006).

Uno de los pilares fundamentales en los que se sustenta el ACP es el análisis de la conducta aplicado (Carr et al., 2002) que ha proporcionado dos contribuciones principales: (a) un marco teórico para la comprensión de las conductas problemáticas y (b) un conjunto de estrategias de evaluación e intervención. En primer lugar, y en relación con los fundamentos teóricos, tanto en la investigación como en la práctica diaria, se ha utilizado el three term contingency (modelo A-C-C, antecedente-conducta-consecuencia; Cooper, Heron y Heward, 2007). Este modelo examina aquellas variables del entorno que provocan y refuerzan las conductas problemáticas (Steege y Watson, 2009), entendiendo que la ocurrencia de la conducta problemática está influenciada por unos antecedentes (estímulos discriminativos) y reforzada por unos eventos (consecuentes) que suceden inmediatamente después de la conducta. No obstante, en las últimas décadas se ha considerado una nueva tipología de antecedentes (eventos situacionales) que no están asociados a la inmediatez de la conducta, pero que tienen un impacto sobre el three-term contingency (Filter y Álvarez, 2011). En este sentido, teniendo en cuenta los eventos situacionales no solo se enfatiza la importancia de los antecedentes en la manifestación de las conductas problemáticas (Steege y Watson, 2009), sino que se puede comprender de manera más amplia la naturaleza de dichas conductas y, en consecuencia, elaborar un análisis más exhaustivo de la conducta (Kubick y McLoughlin, 2010).

En segundo lugar, la aplicación del ACP ha desencadenado el desarrollo de estrategias de evaluación que nos permitan comprender la naturaleza de las conductas problemáticas y diseñar así planes de intervención adecuados a las necesidades de los alumnos (Carr et al., 2002; Riffle, 2011). Entre dichas estrategias, la evaluación funcional de la conducta consiste en un conjunto de procedimientos que permiten identificar y describir las posibles relaciones existentes entre las características de la persona y aquellas variables que propician la manifestación de las conductas o las refuerzan (Steege y Watson, 2009). La literatura nos informa de que la evaluación funcional indirecta permite identificar aquellos eventos que rodean y refuerzan la conducta objeto de estudio (Cooper et al., 2007) utilizando escalas, cuestionarios o entrevistas que se administran a informantes familiarizados con la persona y la conducta problemática (Riffel, 2011). La principal desventaja de esta última estrategia 
recae en que depende de la opinión y el recuerdo del informador, aunque requiere de menos tiempo, menos experiencia por parte de los profesionales y permite, de forma rápida y simple, obtener una primera visión de las posibles relaciones contextuales asociadas a la conducta evaluada (Kelly, LaRue, Roane y Gadaire, 2011).

Actualmente disponemos de estudios recientes que evidencian el uso de estos instrumentos de evaluación funcional indirecta antes de plantear una intervención (p. e., Campbell y Anderson, 2011; Todd, Campbell, Meyer y Horner, 2008), aunque son pocos los estudios que utilizan dichos instrumentos para comprender qué tipo de antecedente está asociado a la ocurrencia de la conducta problemática en un contexto determinado. Por ejemplo, McGill, Teer, Rye y Hughes (2005) exploraron los eventos de antecedentes relacionados con la ocurrencia de las conductas problemáticas que presentan las personas con DI en entornos residenciales. Con dicho propósito, administraron la Entrevista Ecológica (Barratt et al., 2012; McGill et al., 2005) a los profesionales que atendían a 22 personas con DI y que presentaban como mínimo una conducta problemática. Los resultados identifican determinados eventos altamente relacionados con la ocurrencia de la conducta como pueden ser los estados emocionales de las personas (p. ej., estar de mal humor o ansioso), así como antecedentes relacionados con la actividad diaria (p. ej., cuando no se hace nada o cuando se presentan actividades difíciles). Por otro lado, más recientemente, Embregts, Didden, Schreuder y Huitink (2009) utilizaron una adaptación del Inventario de Evaluación de la Conducta (McAtee, Carr y Schulte, 2004) para explorar las variables contextuales que influenciaban las conductas agresivas de 87 personas con DI. Los resultados de su estudio mostraron que las variables sociales/culturales y las relacionadas con las tareas o actividades solían propiciar la manifestación de las conductas problemáticas. En particular, antecedentes como "ser corregido durante una tarea", "actitudes negativas de los profesionales", "tareas difíciles” y "cambios en la rutina”, entre otros, fueron los más significativos.

En línea con dichos estudios, parece imprescindible explorar los eventos que pueden estar asociados a la ocurrencia de las conductas problemáticas, a fin de crear entornos más preventivos y seguros para el alumnado y sistemas educativos que se ajusten a las necesidades conductuales de todos los alumnos (Mansell y Beadle-Brown, 2012). Así, este estudio pretende explorar e identificar aquellas variables contextuales más asociadas a la ocurrencia de las conductas problemáticas que presenta el alumnado de educación primaria de una escuela de educación especial.

\section{Método}

\subsection{Participantes}

El único criterio de inclusión para participar en este estudio era que el alumnado de educación primaria de la escuela presentara alguna conducta desafiante. En total han participado 17 alumnos. Los participantes fueron principalmente niños $(70.6 \%)$ y niñas $(29.4 \%)$ de 6 a 12 años de edad $(M=8.58$; $S D=1.53)$. El grado de disminución

(C) Ediciones Universidad de Salamanca / CC BY-NC-ND

Siglo Cero, vol. 47 (4), n. ${ }^{\circ}$ 260, 2016, octubre-diciembre, pp. 45-56 
de los participantes incluye del $33-64 \%(n=2)$, del $65-74 \%(n=9)$ y mayor del $75 \%$ $(n=6)$.

Considerando que el IEC debe ser contestado pensando en una única conducta problemática, en total se evaluaron 25 conductas de los 17 participantes. Las conductas problemáticas evaluadas incluyen agresión física $(n=10)$, agresión verbal $(n=$ $10)$, conducta autolesiva $(n=4)$, destrucción de la propiedad $(n=2)$, rabietas $(n=2)$, conducta estereotipada $(n=1)$, conducta disruptiva $(n=1)$ y desobediencia $(n=1)$.

Nueve profesionales de atención directa participaron en la recogida de la información relacionada con los participantes. El único criterio que dichos profesionales debían cumplir para participar en el estudio fue haber conocido al alumno a evaluar por un periodo mínimo de 6 meses.

\subsection{Instrumento}

El "Inventario de Evaluación del Contexto" (IEC) (Contextual Assessment Inventory; Carr, Ladd y Schulte, 2008; McAtee et al., 2004) es un instrumento de evaluación funcional indirecta que permite determinar las variables de antecedentes asociadas con la ocurrencia de las conductas problemáticas. Este instrumento está constituido por 80 preguntas, diferenciadas en cuatro categorías (y sus respectivas subcategorías): variables sociales/culturales (interacciones negativas y decepciones), naturaleza de la tarea (factores relacionados con la tarea o la actividad y rutina), variables físicas (entorno incómodo y cambios en el entorno) y variables biológicas (medicación, enfermedad y factores fisiológicos). El instrumento se puntúa mediante una escala de frecuencia Likert, entendiendo que $1=$ nunca y $5=$ siempre. Además, se añaden 13 preguntas abiertas en las que se deben especificar otros posibles eventos que propician la conducta problemática evaluada. Se estima que la duración para contestar el cuestionario es de unos 25 minutos.

En cuanto a las propiedades psicométricas de este instrumento, el estudio de McAtee et al. (2004) indica una alta consistencia interna, mediante el estimador alfa de Cronbach, tanto en su totalidad (.95), como en todas sus categorías: .91, .91, .78 y .57 (social/cultural, naturaleza de la tarea, variables físicas y variables biológicas, respectivamente). Los resultados de un estudio más reciente (Embregts et al., 2009) verifican la consistencia interna del IEC con el mismo estimador en su totalidad (.95) y en sus categorías (rango $=.75, .93$ ). La fiabilidad test-retest y entre observadores del IEC también ha sido evaluada. A pesar de que los resultados de la fiabilidad entre observadores han sido pobres en la mayoría de los ítems, el IEC presenta una fiabilidad aceptable mediante test-retest $(r>.60 ;$ McAtee et al., 2004). Finalmente, la validez de criterio ha sido comprobada con 17 usuarios (Carr et al., 2008). Para este estudio, se ha utilizado la versión validada en nuestro territorio del inventario (Simó-Pinatella, Alomar-Kurz, Font-Roura y Giné, 2012), cuyos resultados indican una alta consistencia (alfa de Cronbach) interna tanto en todo el instrumento (.94) como en sus distintas categorías (social/cultural: .88; naturaleza de la tarea: .88; variables físicas: .79; variables biológicas: .74). 


\subsection{Procedimiento}

La primera autora de este estudio, directora de la escuela Jeroni de Moragas de AMPANS, pidió a los tutores y educadores que trabajan en el centro identificar a aquellos alumnos que presentan conductas problemáticas. Una vez recibida la lista de dichos alumnos, conjuntamente con la psicóloga del centro (cuarta autora) verificaron si dichas conductas se podían considerar conductas problemáticas o no según la conceptualización de Emerson y Einfeld (2011). Se identificó el listado de alumnos que cumplían el criterio de inclusión para este estudio, así como las conductas que presentaban, y se procedió a pedir el consentimiento a las familias para autorizar a los profesionales a dar información sobre los participantes del estudio.

El IEC fue administrado por el segundo y cuarto autor en formato entrevista al tutor y/o educador de referencia. Antes de completar el IEC, se recordaron los objetivos del estudio, se proporcionó información acerca de la estructura y finalidad del instrumento y se pidió al entrevistado rellenar el consentimiento informado. A lo largo de la administración del instrumento, todas las posibles dudas acerca de algún ítem fueron clarificadas por el entrevistador. Con el fin de explorar el acuerdo entre observadores, el IEC fue administrado a un segundo profesional. En total se evaluaron 20 conductas problemáticas.

\subsection{Análisis de datos}

El análisis de los datos cumple dos objetivos específicos. En primer lugar, se calculó el acuerdo entre informadores mediante el índice Kappa, pues es uno de los estimadores de concordancia más robustos al tener en cuenta los acuerdos que se puedan deber al azar. Posteriormente, se analizó la asociación de cada antecedente con la ocurrencia de las conductas evaluadas. Se calcularon las medias y desviaciones típicas de cada subdimensión y se identificaron aquellos ítems en los que la frecuencia de los antecedentes era evaluada con un 4 (casi siempre) o un 5 (siempre) al menos en un $40 \%$ de las ocasiones, en línea con el estudio realizado por Embregts y colaboradores (2009), e indicando que el evento antecedente siempre o casi siempre se relacionaba con la conducta evaluada.

En segundo lugar, se exploró la distribución de los antecedentes en función de la tipología de conducta problemática, tomando como unidad de análisis las diferentes conductas registradas. Dada la infrarrepresentación de las nueve categorías de conductas, se agruparon dichas conductas en cuatro categorías para fines analíticos: (1) agresión (agresión física, agresión verbal y destrucción de la propiedad; Rojahn, Matson, Lott, Esbensen y Smalls, 2001), (2) autolesión, (3) conducta estereotipada y (4) conductas inapropiadas (rabietas, conducta disruptiva, conducta verbal inapropiada y desobediencia; Lowe et al., 2007). Se usaron pruebas estadísticas no paramétricas para estudiar las diferencias entre las dimensiones del IEC en función de la tipología de conducta presentada por los participantes mediante la prueba para muestras independientes de Kruskall Wallis. 


\section{Resultados}

Los resultados apuntan a un grado de concordancia entre los dos informantes entre moderado y bueno en todas las dimensiones de antecedentes, con índices de acuerdo que varían de 0.45 a 0.77 ; y entre moderado y muy bueno en las subdimensiones, con índices de acuerdo que oscilan entre 0.42 y 0.82 . En concreto, el grado de acuerdo más elevado fue en torno a la dimensión "variables biológicas" $(k=0.77)$, seguida de la dimensión "variables físicas" $(k=0.58)$, "social/cultural" $(k=0.50)$ y por último los antecedentes relacionados con la naturaleza de la actividad $(k=0.45)$. En relación a las subdimensiones, el mayor grado de acuerdo entre informantes se halló en los antecedentes relacionados con la "medicación" $(k=0.82)$ y la "enfermedad" $(k=0.82)$, resultados esperables dado que estas dos subdimensiones no dan pie a interpretaciones subjetivas o contextuales de la conducta de los alumnos, sino que se refieren a variables personales más constantes que los educadores conocen (e. g., saber si ha habido cambios en la medicación). Al contrario, la subdimensión en la que los informantes mostraron un mayor desacuerdo fue aquella relacionada con los antecedentes referidos a la "rutina diaria" $(k=0.42)$. Esta subdimensión está inevitablemente más sujeta a interpretaciones de la conducta contextuales y que se pueden ver afectadas por los diferentes momentos del día, situaciones y contextos en los que el informador se haya basado para evaluar los antecedentes relacionados con las conductas evaluadas.

En cuanto a las puntuaciones obtenidas en las cuatro dimensiones, los resultados indican puntuaciones más elevadas en las dimensiones "social/cultural" $(M=2.17 ; S D$ $=1.46)$ y "naturaleza de la actividad" $(M=2.22 ; S D=1.48)$. En concreto, el tipo de antecedente de la dimensión "social/cultural" más asociado con la ocurrencia de la conducta se refiere a las "decepciones" del alumno $(M=2.41 ; S D=1.54)$, mientras que en lo que atañe a la "naturaleza de la actividad", los antecedentes que preceden a la conducta

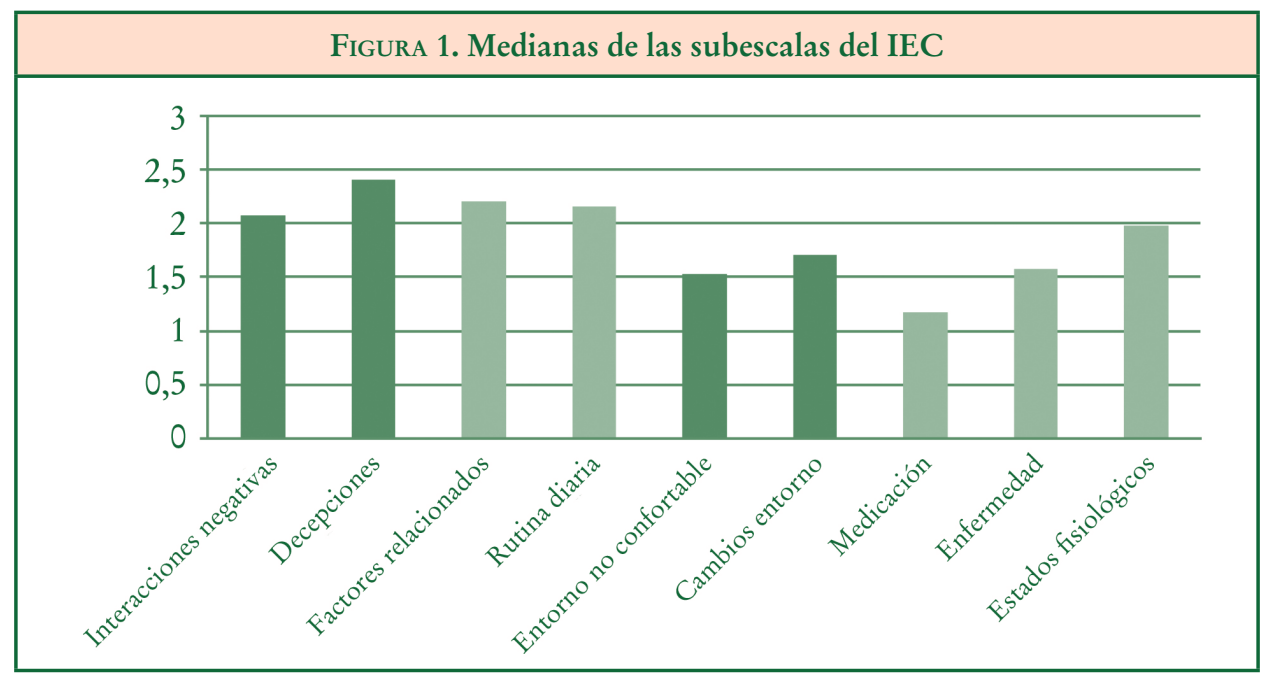

(C) Ediciones Universidad de Salamanca / CC BY-NC-ND

Siglo Cero, vol. 47 (4), n. ${ }^{\circ}$ 260, 2016, octubre-diciembre, pp. 45-56 
desafiante con más frecuencia fueron los "factores relacionados" con la tarea en cuestión $(M=2.2 ; S D=1.47)$. En relación con las "variables físicas” del entorno $(M=1.62$; $S D=1.2)$, los "cambios en el entorno" $(M=1.71 ; S D=1.09)$ se asocian en mayor medida con la ocurrencia de las conductas problemáticas. Los “estados fisiológicos” de los alumnos $(M=1.98 ; S D=1.43)$ son los antecedentes relacionados con las "variables biológicas" de la persona $(M=1.72 ; S D=1.32)$ que suelen anteceder a la ocurrencia de conductas. La Figura 1 ilustra los eventos que en mayor medida suelen anteceder a la ocurrencia de las conductas problemáticas.

Los antecedentes más relacionados con la ocurrencia de las conductas problemáticas corresponden principalmente a variables sociales y culturales $(53.3 \%)$ y en menor medida a aquellos aspectos relacionados con la naturaleza de las actividades (20\%), a las variables físicas del entorno (13.3\%) y a las biológicas (13.3\%) (ver Tabla 1). En concreto, las demandas no atendidas del alumnado son el antecedente que suele preceder en mayor medida a la ocurrencia de la conducta (64\%). Ser castigado por razones conductuales $(60 \%)$ y tener que esperar entre o durante actividades $(60 \%)$ son las situaciones que más se han relacionado con la ocurrencia de la conducta en el centro educativo.

\begin{tabular}{|l|c|}
\hline \multicolumn{2}{|c|}{ TABLA 1. Antecedentes más relacionados con la ocurrencia } \\
de las conductas problemáticas \\
\hline Ítems & Porcentaje (\%) \\
\hline $\begin{array}{l}\text { Social/Cultural } \\
\text { No consigue tener atendidas sus demandas. }\end{array}$ & 64 \\
\hline Ha sido castigado o reñido recientemente por la conducta. & 60 \\
\hline $\begin{array}{l}\text { Ha tenido un desacuerdo o discusión verbal con la familia, profesiona- } \\
\text { les o compañeros. }\end{array}$ & 52 \\
\hline Tiene un mal día en el servicio de actividad de día o en el trabajo. & 48 \\
\hline Compañeros enfadados o que molestan están cerca de él. & 44 \\
\hline El tono de voz de los profesionales es agudo, severo, etc. & 40 \\
\hline Finaliza una actividad preferida. & 40 \\
\hline No tiene comida en momentos deseados. & 40 \\
\hline $\begin{array}{l}\text { Naturaleza de la tarea o de la actividad } \\
\text { Tiene que esperar (por ej., en la fila, sala de espera, etc.). }\end{array}$ & 60 \\
\hline Las tareas son aburridas o no le gustan. & 44 \\
\hline Las tareas son difíciles. & 40 \\
\hline $\begin{array}{l}\text { Variables físicas } \\
\text { Está en relación al momento del día (por ej., amanecer, después de co- } \\
\text { mer, anochecer...). }\end{array}$ & 44 \\
\hline El ambiente es ruidoso. & 40 \\
\hline $\begin{array}{l}\text { Variables biológicas } \\
\text { Padece una enfermedad/dolor agudo (por ej., otitis, estreñimiento, do- } \\
\text { lor de espalda....). }\end{array}$ & 44 \\
\hline Tiene hambre o sed. & 40 \\
\hline
\end{tabular}

() Ediciones Universidad de Salamanca / CC BY-NC-ND

Siglo Cero, vol. 47 (4), n. ${ }^{\circ} 260,2016$, octubre-diciembre, pp. 45-56 
Finalmente, no se hallaron diferencias estadísticamente significativas en la distribución de las dimensiones de antecedentes en función de las tipologías de conducta. La distribución de la dimensión "social/cultural” ( $p=.278)$, "naturaleza de la actividad” ( $p=.082)$, “variables físicas” ( $p=.195)$ y "variables biológicas” $(p=.404)$.

\section{Discusión}

En las últimas décadas, importantes progresos se han realizado para la comprensión, evaluación y tratamiento de las conductas problemáticas (Matson, 2012; Sturmey y Didden, 2014). Este estudio, nos permite seguir avanzando en esta dirección, explorando e identificando aquellas variables de antecedentes más asociadas a la ocurrencia de las conductas problemáticas que presentan los alumnos de educación primaria de una escuela de educación especial.

De acuerdo con el objetivo principal de este estudio, es importante destacar que los resultados identifican las dimensiones referidas a las "variables sociales/culturales" y a la "naturaleza de la tarea o actividad" como aquellas altamente relacionadas con la ocurrencia de las conductas evaluadas. Concretamente, las subcategorías "decepciones" y "factores relacionados con la actividad" son las que reciben mayores puntuaciones. Por el contrario, las variables referentes al "entorno físico" y "factores biológicos” parecen tener menor relación con dichas conductas. En este caso, las subcategorías con menor relación con la ocurrencia de las conductas evaluadas hacen referencia a "medicación” y "entorno no confortable". Finalmente, en este estudio no se encontraron diferencias significativas entre la tipología de las conductas problemáticas y las distintas variables de antecedentes.

A pesar de que la investigación en esta temática es escasa, los resultados de nuestro estudio concuerdan con trabajos previos. Por ejemplo, Embregts et al. (2009) identificaron las variables "sociales/culturales" y de la "naturaleza de la tarea o actividad" como las más asociadas a las conductas problemáticas. De manera parecida, aunque mediante otro instrumento, McGill y colaboradores (2005) identificaron determinados antecedentes (p. e., relacionados con la actividad diaria) como aquellos más asociados con la ocurrencia de dichas conductas.

A pesar de las aportaciones que plantea este estudio, es importante destacar algunas limitaciones. En primer lugar, con el fin de identificar aquellos eventos más asociados con la ocurrencia de las conductas problemáticas se ha utilizado un instrumento de evaluación funcional indirecta. En este sentido, hubiera sido interesante contrastar la opinión de los informantes mediante observaciones directas (es decir, mediante una evaluación funcional directa). Segundo, debido a la infrarrepresentación de algunas de las tipologías de conductas se aglomeraron algunas de ellas en cuatro categorías diferentes. Si bien dicha decisión se alinea con categorizaciones propuestas a nivel teórico (por ej., Lowe et al., 2007; Rojahn et al., 2001), se pierde información sobre la relación de cada tipología de conducta específica con las diferentes variables de antecedentes. Finalmente, al haber hecho uso de técnicas de contraste no paramétricas, menos robustas que las paramétricas, las conclusiones del estudio deben interpretarse con cautela y de forma contextualizada. 
La comprensión de las conductas problemáticas resulta compleja. Se han propuesto distintos modelos para comprender la naturaleza de dichas conductas (Matson et al., 2011) y algunos estudios (p. e., Didden et al., 2012) subrayan que las conductas problemáticas pueden ser el resultado de la interacción con el contexto. Es imperativo, pues, invertir en estrategias de intervención que se identifiquen por su carácter esencialmente preventivo y proactivo como, por ejemplo, modificando los antecedentes (Bambara y Knoster, 2009; Smith, 2011). Es decir, cambiar, alterar o eliminar aquellos eventos o estímulos del entorno que suceden antes de la conducta problemática y propician su ocurrencia (Filter y Álvarez, 2011), reduciendo así no sólo la ocurrencia de la conducta problemática (Bambara y Knoster, 2009), sino también las intervenciones que preceden a la ocurrencia de dicha conducta y que habitualmente se han centrado en el castigo (Snell, 2010).

Desde esta perspectiva, se pueden plantear algunas implicaciones prácticas y en relación a futuras investigaciones. En cuanto a las implicaciones prácticas, es necesario invertir en la prevención de la conducta problemática del alumnado. Es decir, debemos analizar las características de nuestros centros educativos y de nuestras prácticas con el fin de identificar aquellos eventos antecedentes que preceden y ocasionan la ocurrencia de las conductas problemáticas (Luiselli, 2006). Además, proporcionar la oportunidad de enseñar habilidades alternativas y construir entornos y condiciones reforzantes tanto para la persona como para el profesional (Bambara y Knoster, 2009). En segundo lugar, la evaluación funcional indirecta es una estrategia altamente eficaz para comprender la conducta evaluada, así como para realizar intervenciones ajustadas. Es más probable que una intervención sea efectiva y responda a las necesidades conductuales de la persona que las manifiesta cuando se da respuesta a aquello que mantiene la conducta y, a la vez, se modifican aquellos antecedentes que promueven dicha ocurrencia.

Finalmente, es necesario explorar las relaciones que se establecen entre las características personales del alumnado y las variables contextuales (Medeiros, Rojahn, Moore y van Ingen, 2014). Resulta ineludible seguir avanzando en la elaboración de instrumentos que nos permitan proporcionar entornos más preventivos, activos y ecológicos. Este estudio, así como aquellos que han pretendido identificar aquellos eventos relacionados con la ocurrencia de las conductas (Embregts et al., 2009; McGill et al., 2005), han utilizado un instrumento de evaluación funcional indirecta pensado para la evaluación de los antecedentes relacionados con la conducta de un individuo, con el fin de identificar los antecedentes a nivel de centro. Estas iniciativas ponen de relieve la necesidad de plantear y desarrollar instrumentos universales que permitan una evaluación directa del contexto a fin de identificar aquellos entornos/ situaciones de antecedentes altamente relacionados con la ocurrencia de las conductas desafiantes para todo el alumnado.

\section{Referencias bibliográficas}

Bambara, L. M. y Knoster, T. P. (2009). Designing Positive Behavior Support Plans (2. a ed.). Washington: AAIDD.

(C) Ediciones Universidad de Salamanca / CC BY-NC-ND

Siglo Cero, vol. 47 (4), n. ${ }^{\circ}$ 260, 2016, octubre-diciembre, pp. 45-56 
Barrat, N., McGill, P. y Hughes, C. (2012). Antecedent influences on challenging behavior: a preliminary assessment of reliability, generalisability and validity of the Ecological Interview. BILD, International Journal of Positive Behavioural Support, 2, 2, 31-41.

Bradshaw, C. P., Reinke, W. M., Brown, L. D., Bevans, K. B. y Leaf, P. J. (2008). Implementation of School-wide positive behavioral interventions and supports (PBIS) in elementary schools: observations from randomized trials. Education and Treatment of Children, 31, 1-26.

Campbell, A. y Anderson, C. M. (2011). Check-in/Check-out: a systematic evaluation and component analysis. Journal of Applied Behavior Analysis, 44, 315-326.

Carr, E. G., Dunlap, G., Horner, R. H., Koegel, R. L., Turnbull, A. P., Sailor, W., ... y Fox, L. (2002). Positive behavior support evolution of an applied science. Journal of positive behavior interventions, 4 (1), 4-16.

Carr, E. G., Ladd, M. V. y Schulte, C. F. (2008). Validation of the Contextual Assessment Inventory for problem behavior. Journal of Positive Behavior Interventions, 10, 91-104.

Cooper, J. O., Heron, T. E. y Heward, W. L. (2007). Applied behavior analysis (2. ${ }^{\text {ed.). Up- }}$ per Saddle River, NJ: Pearson Education Inc.

Crone, D. A. y Horner, R. H. (2003). Building positive behavior support systems in schools: Functional behavioral assessment. Guilford Publications.

Didden, R., Sturmey, P., Sigafoos, J., Lang, R., O’Reilly, M. F. y Lancioni, G. E. (2012). Nature, prevalence, and characteristics of challenging behavior. En J. J. Matson (Ed.), Functional assessment of challenging behaviors (pp. 23-44). New York: Springer.

Embregts, P. J., Didden, R., Schreuder, N. y Huitink, C. (2009). Contextual variables affecting aggressive behavior in individuals with mild to borderline intellectual disabilities who live in a residential facility. Journal of Intellectual Disability Research, 53, 255-264.

Emerson, E. y Einfeld, S. T. (2011). Challenging behavior (3rd ed.). Cambridge, UK: Cambridge University Press.

Filter, K. J. y Álvarez, M. E. (2011). Functional behavior assessment. A three-tired prevention model. New York: Oxford.

Kelley, M. E., La Rue, R. H., Roane, H. S. y Gadaire, D. M. (2011). Indirect behavioral assessments. Interviews and rating scales. En W. W. Fisher, C. C. Piazza y H. S. Roane (Eds.), Hanbook of applied behavior analysis (pp. 182-190). New York: The Guilford Press.

Kubick, R. J. y McLoughlin, C. S. (2010). Alternatives to Functional Behavior Assessments as "Educational Autopsies": An Essay Review. Education Review, 2 (13), 1-19.

Lassen, S. R., Steele, M. M. y SAilor, W. (2006). The relationship of school-wide positive behavior support to academic achievement in an urban middle school. Psychology in the Schools, 43, 701-712.

Lecavalier, L., LeOne, S. y Wiltz, J. (2006). The impact of behaviour problems on caregiver stress in young people with autism spectrum disorders. Journal of Intellectual Disability Research, 50, 172-183.

Lowe, K., Allen, D., Jones, E., Brophy, S., Moore, K. y James, W. (2007). Challenging behaviours: Prevalence and topographies. Journal of Intellectual Disability Research, 51, 625-636.

Luiselli, J. K. (2006). Antecedent variables and intervention. Supporting children and adults with developmental disabilities in community settings. Baltimore: Paul H. Brookes.

Mansell, J. y Beadle-Brown, J. (2012). Active support: Enabling and empowering people with intellectual disabilities. Great Britain: Jessica Kingsley Publishers.

MAtson, J. L. (2012). Functional assessment of challenging behaviors. New York: Springer.

Matson, J. L., Kozlowski, A. M., Worley, J. A., Shoemaker, M. E., Sipes, M. y Horovitz, M. (2011). What is the evidence for environmental causes of challenging behaviors in persons

(C) Ediciones Universidad de Salamanca / CC BY-NC-ND

Siglo Cero, vol. 47 (4), n. ${ }^{\circ}$ 260, 2016, octubre-diciembre, pp. 45-56 
with intellectual disabilities an autism spectrum disorders? Research in Developmental Disabilities, 32, 693-698.

McAtee, M., CarR, E. y SChulte, C. (2004). A Contextual Assessment Inventory for problem behavior. Journal of Positive Behavior Interventions, 6, 148-165.

McGill, P., Teer, K., Rye, L. y Hughes, D. (2005). Staff reports of setting events associated with challenging behavior. Behavior Modification, 29, 599-615.

Medeiros, K., Rojahn, J., Moore, L. L. y van Ingen, D. J. (2014). Functional properties of behaviour problems depending on level of intellectual disability. Journal of Intellectual Disability Research, 58, 151-161.

Miller, L. M., Dufrene, B. A., Sterling, H. E., Olmi, D. J. y Bachmayer, E. (2015). The effects of check-in/check-out on problem behavior and academic engagement in elementary school students. Journal of Positive Behavior Interventions, 17, 28-38.

Moreno, G. (2011). Addressing challenging behavior in the general education setting: conducting a teacher-based Functional Behavioral Assessment (FBA). Education, 3-13, 1-9.

Riffel, L. A. (2011). Positive Behavior Support at the Tertiary Level. California: Corwin.

Rojahn, J., Matson, J. L., Lott, D., Esbensen, A. J. y Smalls, Y. (2001). The Behavior Problems Inventory: An instrument for the assessment of self-injury, stereotyped behavior, and aggression/destruction in individuals with developmental disabilities. Journal of $\mathrm{Au}-$ tism and Developmental Disorders, 31, 577-588.

Ross, S. W., Romer, N. y Horner, R. H. (2011). Teacher well-being and the implementation of schoolwide positive behavior interventions and supports. Journal of Positive Behavior Interventions, 14, 118-128.

Simó-Pinatella, D., Alomar-Kurz, E., Font-Roura J. y Giné, C. (2012). Evaluación de los problemas de conducta de las personas con discapacidad intelectual: Resultados de la prueba piloto. En VIII Jornadas Científicas Internacionales de Investigación sobre Discapacidad. Salamanca: Amarú Ediciones.

Simó-Pinatella, D., Alomar-Kurz, E., Font-Roura, J., Giné, C. y McGill, P. (2015). Efectos de las operaciones motivadoras en una conducta autolesiva mediante las presesiones. Escritos de Psicología, 8 (2), 58-65.

SмrTh, R. G. (2011). Developing Antecedent Interventions for Problem Behavior. En W. W. Fisher, C. C. Piazza, y H. S. RoAne (Eds.), Handbook of Applied Behavior Analysis (pp. 297-316). New York: The Guilford Press.

SNeLL, M. (2010). What is positive behaviour support (PBS)? How does PBS promote appropriate behaviour? Conferencia en I Jornades Internacionals sobre discapacitat intel-lectual. Consorci Sant Gregori, Girona. España.

Steege, M. W. y Watson, T. S. (2009). Conducting school-based functional behavioral assessment. A practitioner's guide. 2nd edition. New York: Guilford.

Stormont, M., Lewis, T. J., BeCKNeR, R. y Johnson, N. W. (2008). Implementing positive behavior support systems in Early Childhood and Elementary Settings. California: Corwin Press.

Sturmey, P. y DidDen, R. (2014). Evidence-based practice and intellectual disabilities. West Sussex: John Wiley \& Sons.

Sugai, G. y Horner, R. (2002). The evolution of discipline practices: School-wide positive behavior supports. Child and Family Behavior Therapy, 24, 23-50.

Todd, A. W., Campbell, A. L., Meyer, G. G. y Horner, R. H. (2008). The effects of a targeted intervention to reduce problem behaviors elementary school implementation of check incheck out. Journal of Positive Behavior Interventions, 10, 46-55. 